\title{
Interactions of Phlebopus spongiosus with several soil fungi and antibacterial activity of its culture broth
}

\author{
Pham Nguyen Duc Hoang ${ }^{1}$, Ho Bao Thuy Quyen ${ }^{2 *}$, Akira Suzuki ${ }^{3,4}$ \\ ${ }^{1}$ Institute of Mycology and Biotechnology, Vietnam \\ ${ }^{2}$ Ho Chi Minh City Open University, Vietnam \\ ${ }^{3}$ Tokyo City University, Japan \\ ${ }^{4}$ Agricultural Hi-Tech Park of Ho Chi Minh City, Vietnam \\ *Corresponding author: quyen.hbt@ou.edu.vn
}

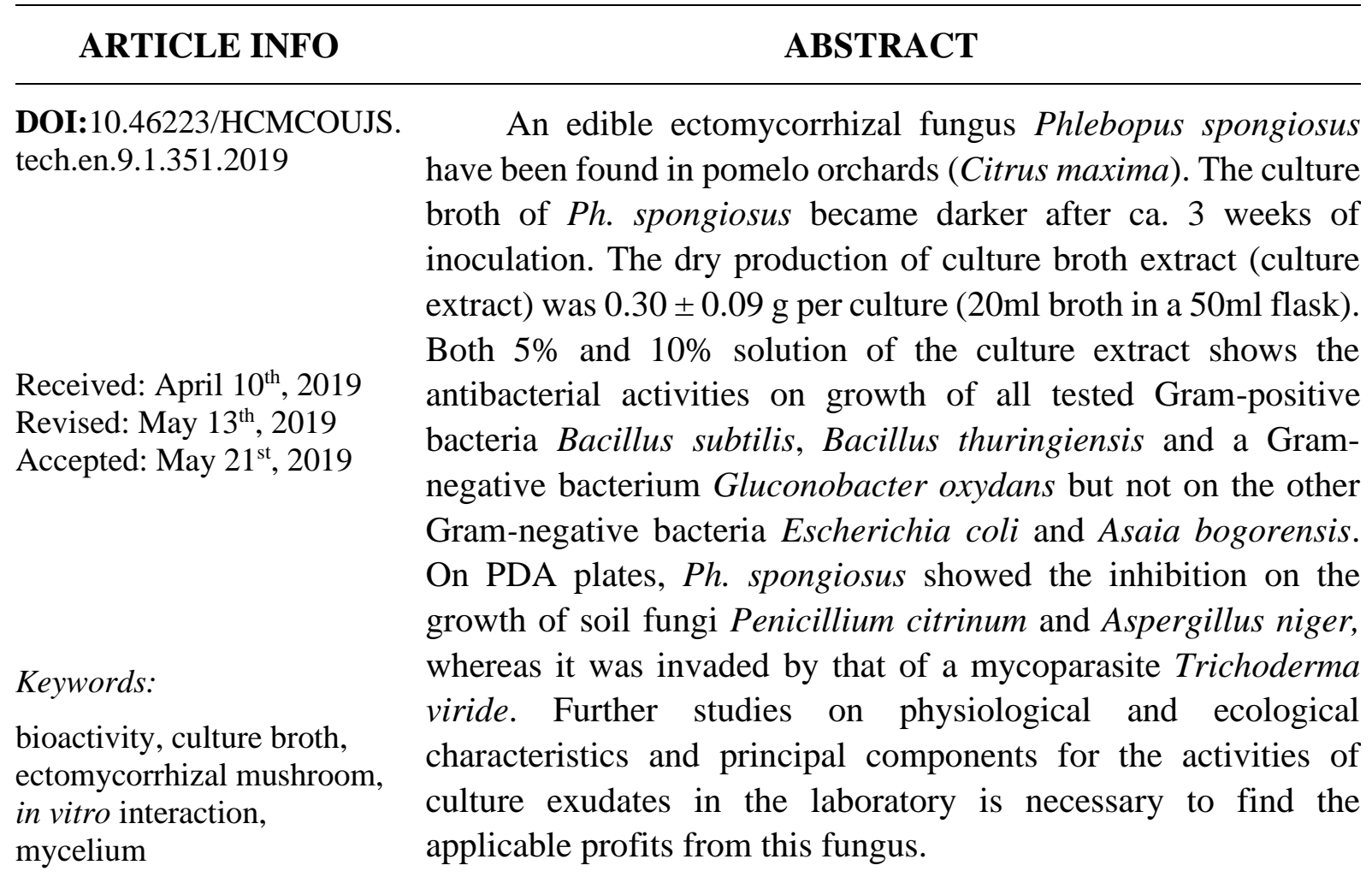

\section{Introduction}

Fungi, especially saprotrophs, also work as a decomposer for recycling the remainder of organisms and supplying nutrient again to other living organisms (Stamets, 2005). They also share the same habitat inside the mycobiota in soil and interact with each other, other microbes, small insects and plants. From the ecological viewpoint, any biological interaction in nature should be placed into several radiate interaction with other organisms which share the same habitat. Largely, interaction physiology has been studied using plate culture in which the two species, usually, or exacting substances of a species and another species are opposed for assessing outcome (Cooke \& Whipps, 1993). The interaction between a fungus - a fungus, with or without other organisms, on both in situ and in vitro has been paid attention by mycologists. By the sequencing publishes of Frankland, Hedger, and Swift (1982), Cooke and Rayner 
(1984), and Woodland and Boddy (2008), several patterns and definitions of fungus - fungus interaction were established.

Besides, fungi look like "the amazing chemical factories" (Wainwright, 2010) which is used to produce a range of commercial products from clothes dyes to life-saving drugs (antibiotic). For a long time from ancient, mushrooms have been considered to have medicinal value. Fungi were assumed to use firstly as "a magic drugs" or a hallucinogen, not as food, from 7000-9000 years ago in Sahara Desert areas (Rutter, 2010). Then in Asia, some fungi were used as a superior healthy supplementary by ancient Chinese in ca. 2000 years ago such as Ganoderma lucidum (Chang \& Miles, 2004). About 700 species of fungi were known medicinal properties and about 1800 species of mushrooms were reckoned to potential medicinal attributes (Chang \& Miles, 2004).

The fungal values usually derive from their exudates or their inside chemical substances. Most are the intra-basidiomata compounds such as cordycepin in Cordyceps sinnesis, garnoderic acid in Ganoderma lucidum or a recent compound ergothioneine in many edible mushrooms (Ohshima, 2011). However, some are the mycelial compounds as a case of krestin (PSK) in Tramates versicolor; mycelial culture broth as schizophyllan in Schizophyllum commune and some from all part of the fungus as the case of Ganoderma lucidum. The example of pharmaceutical values from different origins of some common mushrooms was shown in Table 1 following Chang and Miles (2004).

\section{Table 1}

The example of pharmaceutical values from different origins of some common mushrooms (Chang \& Miles, 2004)

\begin{tabular}{lccc}
\multicolumn{1}{c}{ Species } & $\begin{array}{c}\text { Cultivated } \\
\text { fruiting body }\end{array}$ & Cultivated mycelium & Culture broth \\
\hline Agaricus blazei & ++ & + & + \\
Flammulina velutipes & ++ & + & - \\
Ganoderma lucidum & ++ & + & + \\
Grifola frondosa & + & + & - \\
Hericium erinaceus & ++ & + & + \\
Lentinula edodes & ++ & + & + \\
Schizophyllum commune & - & - & - \\
Tramates versicolor & ++ & + & + \\
Volvariella volvacea & & + & + \\
\hline
\end{tabular}

Note:++ High bioactive effects; + moderate bioactive effects; — not available

Source: The researcher's data analysis 
Phlebopus spongiosus is a terrestrial, edible, ectomycorrhizal fungus and most of its basidiomata have been found in pomelo orchards (Citrus maxima), appearing around the bases of the plants (Pham et al., 2012a, 2012b). In our study, this fungus was investigated several physiological and ecological characteristics in the laboratory as the fundamental data. Some applicable profits from these characteristics would be also recorded from these data.

During the cultivation of $\mathrm{Ph}$. spongiosus, its colonies altered several media, both broth and agar, to darker by the mycelial exudate. The culture broth is seemed to be a suitable candidate for applicable substance(s) from this edible fungus. The extract of culture broth of Ph. spongiosus in Ohta medium was collected and applied to test antibacterial activity. For investigating the relationship between this fungus and other soil fungi, the in vitro interactions of colonies of ectomycorrhizal fungus $P$ h. spongiosus with some common soil fungi Aspergillus niger, Penicillium citrinum and Trichoderma viride were studies from the view of ecology.

\section{Materials and methods}

\section{Organisms}

A mycelial strain of $\mathrm{Ph}$. spongiosus was isolated from a paratype basidioma (specimen voucher CBM FB-38670 deposited in Natural History Museum and Institute, Chiba, Japan) on MMN (Modified Melin and Norkrans) medium (Marx, 1969) and maintained on PDA medium [4g potato extract (Sigma Aldrich, MO, USA), 20g glucose (Wako, Tokyo, Japan), 15g agar (Difco, MI, USA) and filling to 10001 with distilled water] at $20 \pm 1^{\circ} \mathrm{C}$, in darkness.

The bacterial strains were from the National Biological Resource Center, National Institute of Technology and Evaluation (NITE - NBRC), Japan (Table 2). Bacillus subtilis subsp. subtilis, Bacillus thuringiensis and Escherichia coli were maintained on 802 broth (NBRC, NBRC website) [10g peptone (Difco, MI, USA), 2g yeast extract (Difco, MI, USA), $1 \mathrm{~g} \mathrm{MgSO}_{4} .7 \mathrm{H}_{2} \mathrm{O}$ (Wako, Tokyo, Japan) and filling to $1000 \mathrm{ml}$ with distilled water]. Gluconobacter oxydans and Asaia bogorensis were maintained on 804 broth (NBRC, NBRC website) [5g peptone (Difco, MI, USA), $5 \mathrm{~g}$ yeast extract (Difco, MI, USA), $5 \mathrm{~g}$ glucose (Wako, Tokyo, Japan), $1 \mathrm{~g} \mathrm{MgSO}_{4} .7 \mathrm{H}_{2} \mathrm{O}$ (Wako, Tokyo, Japan) and filling to $1000 \mathrm{ml}$ with distilled water]. All were incubated at $30 \pm 1^{\circ} \mathrm{C}$ in darkness.

\section{Table 2}

Bacterial strains used in antibacterial tests

\begin{tabular}{lcc}
\hline \multicolumn{1}{c}{ Bacterial species } & Strain number & Gram stain \\
\hline Escherichia coli & NBRC 3301 & Negative \\
Gluconobacter oxydans & NBRC 14819 & Negative \\
Asaia bogorensis & NBRC 16594 & Negative \\
Bacillus subtilis subsp. subtilis & NBRC 13719 & Positive \\
Bacillus thuringiensis & NBRC 101235 & Positive \\
\hline
\end{tabular}

Source: The researcher's data analysis 
The mycelial strain of $A$. niger (IFM55890), P. citrinum (IFM40616) and T. viride (IFM40938) were from the collection of Research Center for Pathogenic Fungi and Microbial Toxicoses, Chiba University, Japan. All were also maintained on PDA medium at $20 \pm 1^{\circ} \mathrm{C}$, in darkness.

\section{In vitro interactions}

The in vitro interaction was examined on PDA plates $(90 \mathrm{~mm}$ in diameter, $10 \mathrm{~mm}$ in high). Interaction between $P h$. spongiosus with each mold was examined by 5 PDA plates.

A mycelial disk $4 \mathrm{~mm}$ in diameter of Ph. spongiosus was bored out using a cork borer, from the sub-peripheral region of a colony grown on PDA plates, and aseptically transferred to the side of the new PDA plate. All inoculated plates were incubated at $25 \pm 1^{\circ} \mathrm{C}$ in darkness. After 72 hours, each mold (A. niger/P. citrinum/T. viride) was spot inoculated onto the opposite side of the plate. The distance between the two inocula is about $40-50 \mathrm{~mm}$. All plates were again incubated at $25 \pm 1^{\circ} \mathrm{C}$, in darkness.

After several days, the plates were observed to check the interaction. After two colonies contacting together, all plates were also kept a long time until the pattern of fungal interaction became stable. The patterns of interaction were described following Cooke and Rayner (1984).

\section{Culture broth extracting}

A mycelial disk $4 \mathrm{~mm}$ in diameter of Ph. spongiosus was bored out using a cork borer, from sub-peripheral regions of colonies grown on PDA plate, and aseptically transferred into $20 \mathrm{ml}$ of Ohta broth (Ohta, 1990) in a 50ml conical flask (Pyrex Iwaki, Tokyo, Japan), corked with a sterilized Silicosen plug (Shin-Etsu Polymer, Tokyo, Japan).

After about 6 months, the culture broth was aseptically harvested by filtering two times with a glass filter (17G3, Pyrex Iwaki, Tokyo, Japan) and membrane filter (pore size $0.2 \mu \mathrm{m}$, mixed cellulose ester membrane, Advantec, Tokyo, Japan). Then, the culture (20ml of culture broth in a $50 \mathrm{ml}$ flask) broth was condensed by freeze-drying in a sterile condition for collecting culture extract. The extract of each culture was collected and weighted.

\section{Antibacterial activity}

For each bacterium, 5 agar plates of maintaining medium were prepared to test the antibacterial activity of fungal exudates by the diffusion method. The stainless steel penicylinders (peni-cylinders, BioLogis Inc., Virginia, USA) of $6 \mathrm{~mm}$ in the inner diameter, 10mm high were used for diffusing the fungal extract broth on the surface of agar plates.

Each $200 \mu \mathrm{l}$ of a bacterial suspension at a density of ca. $3 \times 10^{6}$ cells $/ \mathrm{ml}$ was aseptically spread on the surface of an agar plate by a glass spreader; the peni-cylinder was then placed in the center of each plate; a $200 \mu 1$ solution was poured into the peni-cylinder; then it was incubated at $30 \pm 1^{\circ} \mathrm{C}$ in darkness, and observed after 24 hours. For the preliminary investigation, the water solutions of broth extract used in this study were 5\% $(50 \mu \mathrm{g}$ extract $/ \mathrm{ml})$ and $10 \%$ $(100 \mu \mathrm{g}$ extract $/ \mathrm{ml})$. The solution of Ohta broth (1x Ohta broth) and 10 times the concentration of Ohta broth (10x Ohta broth) were used as control. 


\section{Results}

\section{In vitro interactions}

After about 48 hours of inoculation, the mycelia of $P h$. spongiosus started to grow from inocula.

After about 3-4 days of inoculation, the colonies of $T$. viride were expanded and contacted with those of $P h$. spongiosus. Then, the mycelia of $T$. viride infiltrated and invaded those of Ph. spongiosus (Figure 1a). Finally, colonies of $T$. viride completely covered those of Ph. spongiosus after 2-3 weeks (Figure 2b).
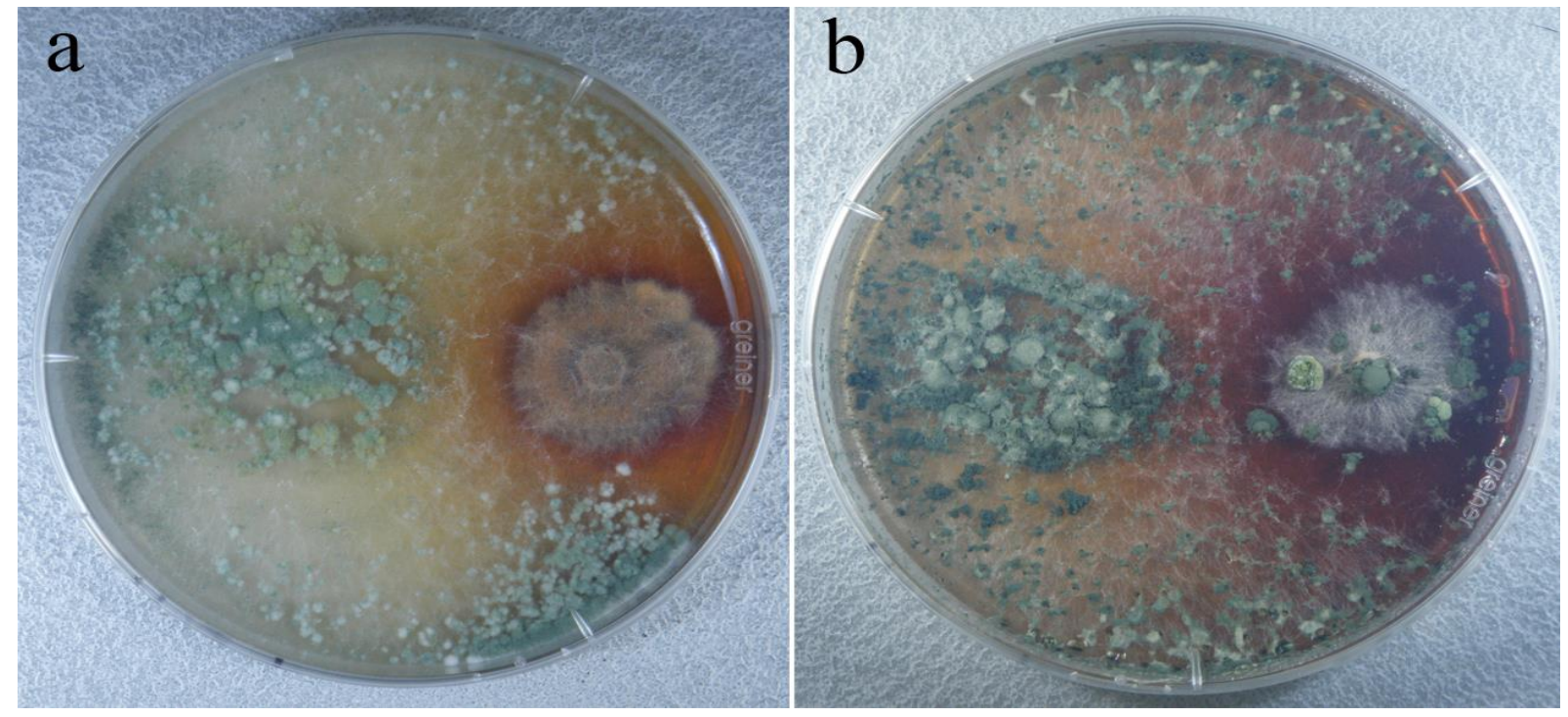

Figure 1. Interaction between Phlebopus spongiosus and Trichoderma viride

Note: a: The mycelium of $T$. viride infiltrated and invaded that of $P$. spongiosus after 5 days of inoculation of the mold;

b: Colony of $T$. viride completely covered that of Ph. spongiosus after 2-3 weeks of inoculation of mold

In cases of $P$. citrinum and $A$. niger, their colonies were expanded and contacted with those of Ph. spongiosus after ca. 1 week. Both of their colony expansions were barred by colonies of Ph. spongiosus as deadlock interaction (Figures 2a, 3a). These deadlock interactions did not change at 10 weeks of inoculation of mold. The interaction between Ph. spongiosus and $A$. niger was the deadlock antagonism and that between $P h$ spongiosus and $P$. citrinum was the deadlock competition. 


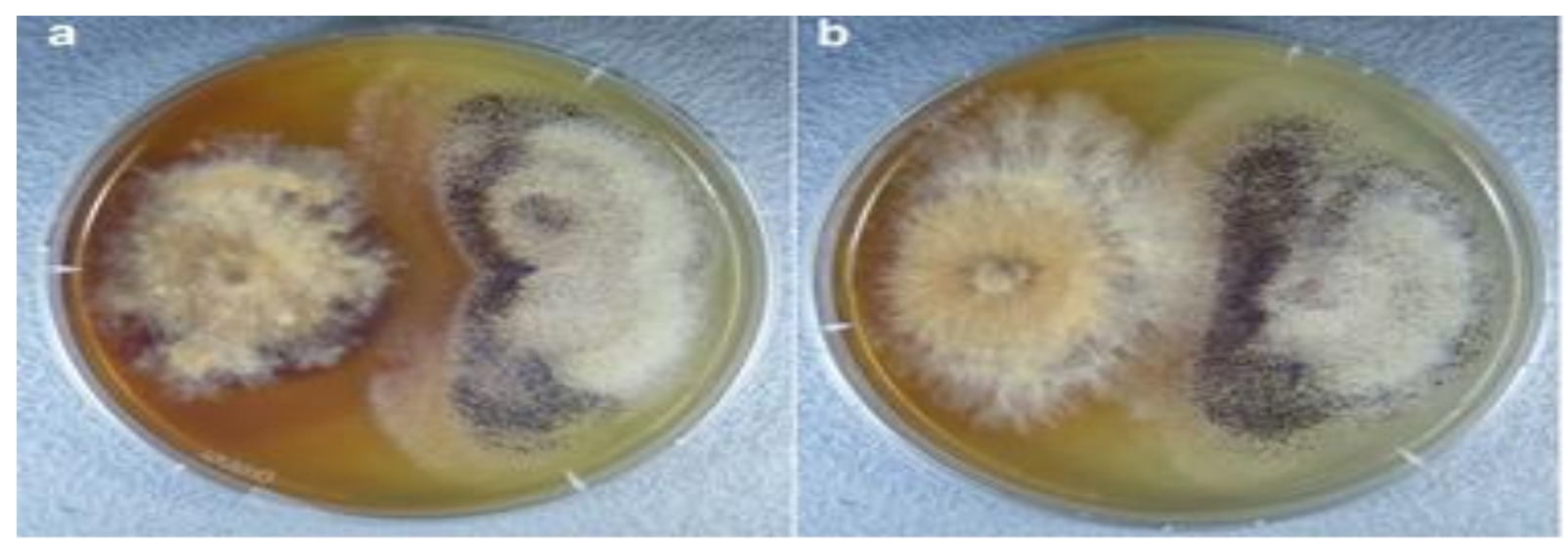

Figure 2. Interaction between Phlebopus spongiosus and Aspergillus niger

Note: a: The deadlock antagonism interaction after 6 weeks of incubation between A. niger and P. spongiosus and had not changed until 10 weeks;

b: The mycelium of Ph. spongiosus invaded and covered that of A. niger after 3 weeks of incubation and had not changed until 10 weeks

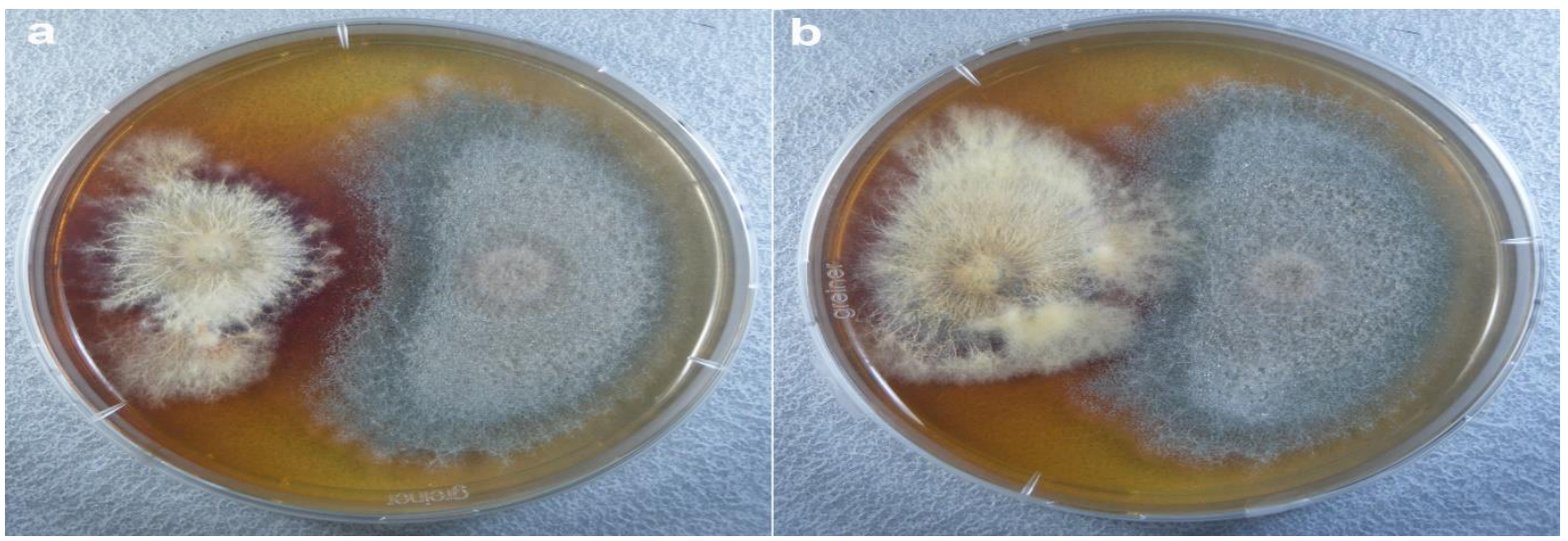

Figure 3. Interaction between Phlebopus spongiosus and Penicillium citrinum

Note: a: The deadlock competition interaction after 6 weeks of incubation between P. citrinum and Ph. spongiosus and had not changed until 10 weeks;

b: The mycelium of $P h$. spongiosus invaded and covered that of $P$. citrinum after 3 weeks of incubation and had not changed until 10 weeks

However, after 2-3 weeks, colonies of Ph. spongiosus in some plates invaded and covered those of $A$. niger and $P$. citrinum (Figures $2 \mathrm{~b}, 3 \mathrm{~b}$ ). These invasion interactions were also not changed at 10 weeks of inoculation of mold.

\section{Culture broth extract}

After a few days of inoculation, mycelia started to expand from inoculum in all broths. Fungal colonies secreted exudates which blackened the medium after ca. 3 weeks. The culture broth changed to pure black after about 4 months (Figures $4 b, 5 a$ ). In a long time of incubation, $P h$. spongiosus formed primordia in both agar plate and broth, especially in Ohta medium (Figure 4). 

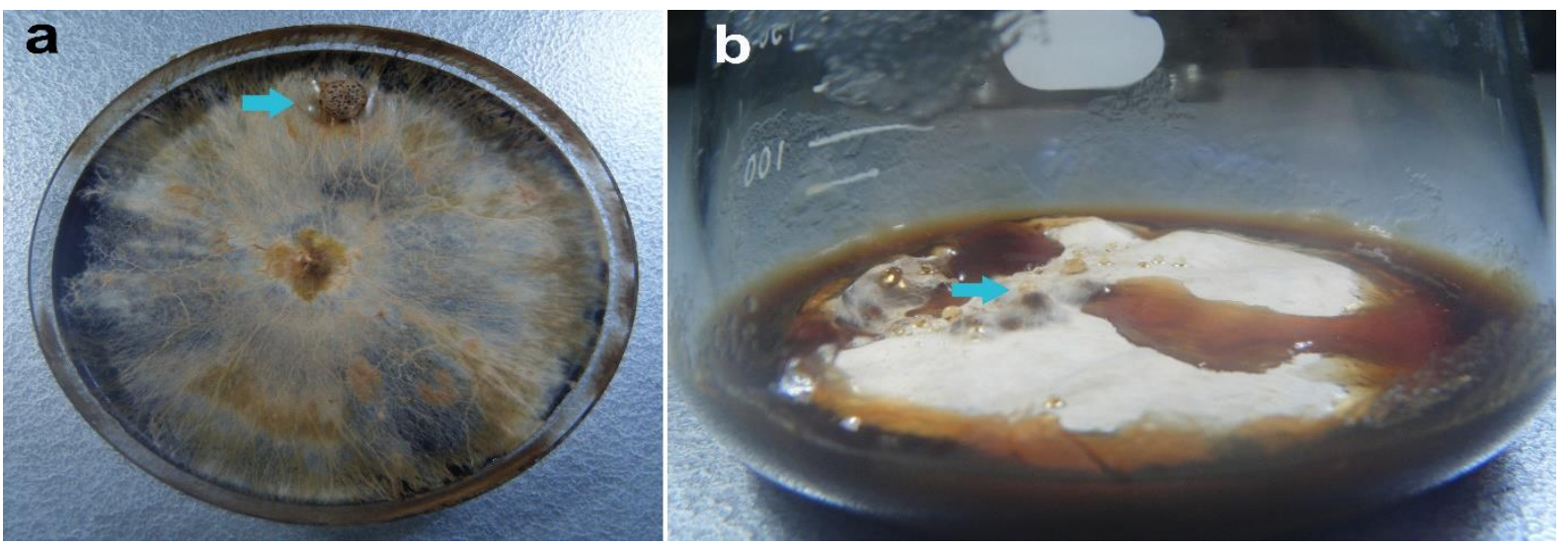

Figure 4. Cultivation of Phlebopus spongiosus in Ohta medium. Blue arrows indicated primordia

Note: a: On Ohta plates; b: In Ohta broth

The culture broth extract (Figure $5 \mathrm{~b}$ ) production was $0.30 \pm 0.09 \mathrm{~g}$ per $20 \mathrm{ml}$ culture broth. This extract was pure black, glutinous and soluble in water but insoluble in alcohol $95 \%$.

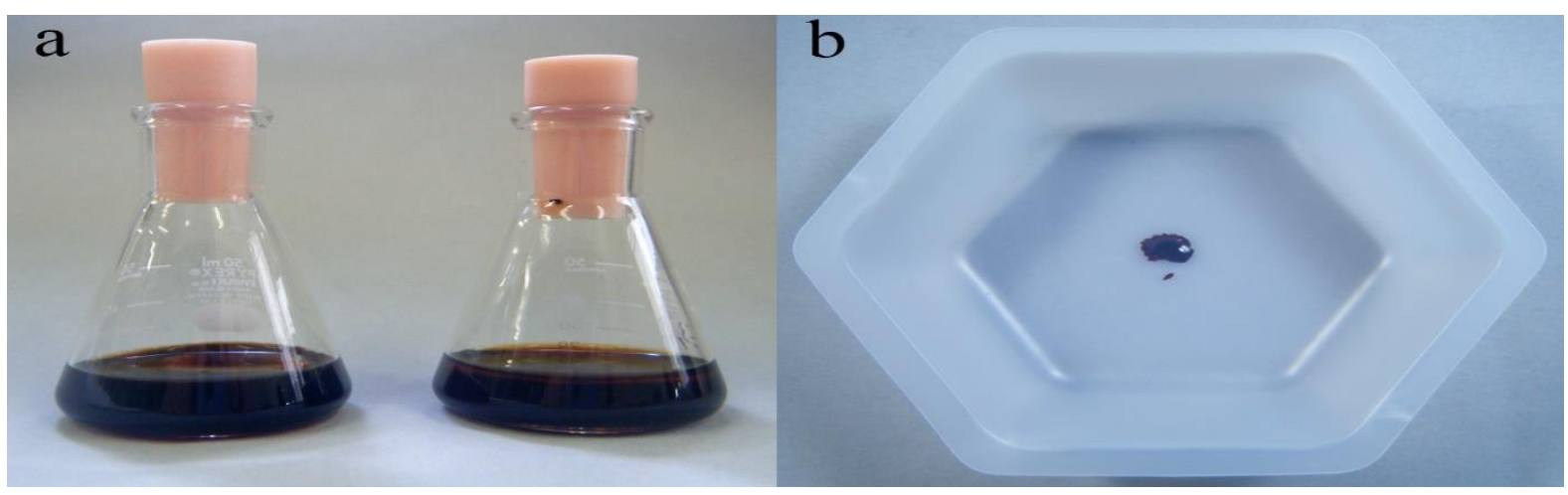

Figure 5. Culture broth and broth extract of Phlebopus spongiosus

Note: a: Fungal colonies of Ph. spongiosus blacked Ohta medium after 4 moths of inoculation; b: Extract of culture broth from Ph. spongiosus culture on Ohta medium

\section{Antibacterial activity}

The solutions of 1x Ohta broth and 10x Ohta broth have no effect in growth of all bacteria. However, these solutions of Ohta broth changed the pattern of Bacillus colonies, especially on B. subtilis, to more transparency comparing with the normal pattern (Figure 6). The 5\% and $10 \%$ solutions of broth extract have no effect on the growth of Gram-negative $E$. coli and A. bogorensis (Figure 7). The 5\% and $10 \%$ solutions of broth extract show the inhibition of the growth of Gram-positive B. thuringiensis, B. subtilis and Gram-negative $G$. oxydans (Figures 6,7). The clear zone diameters in all inhibited effects are about $1-2 \mathrm{~cm}$. 

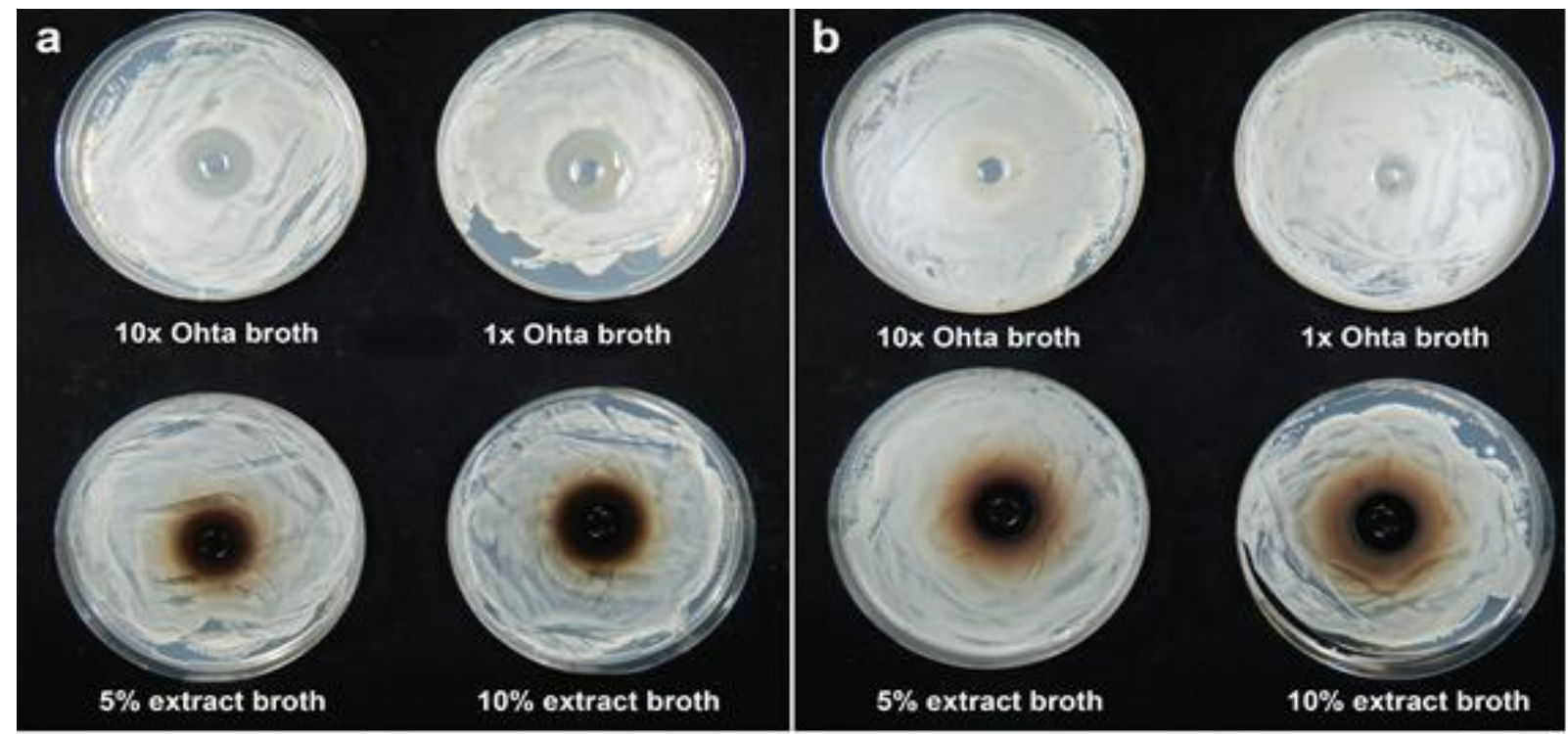

Figure 6. Antibacterial activities of fungal culture broth extract in Gram-positive bacteria. The inside circle is derived from a contact of peni-cylinder on the surface of the plate; the outer circle indicates the clear zone

Note: a: The inhibited effect on the growth of Bacillus subtilis; b: The inhibited effect on the growth of Bacillus thuringiensis

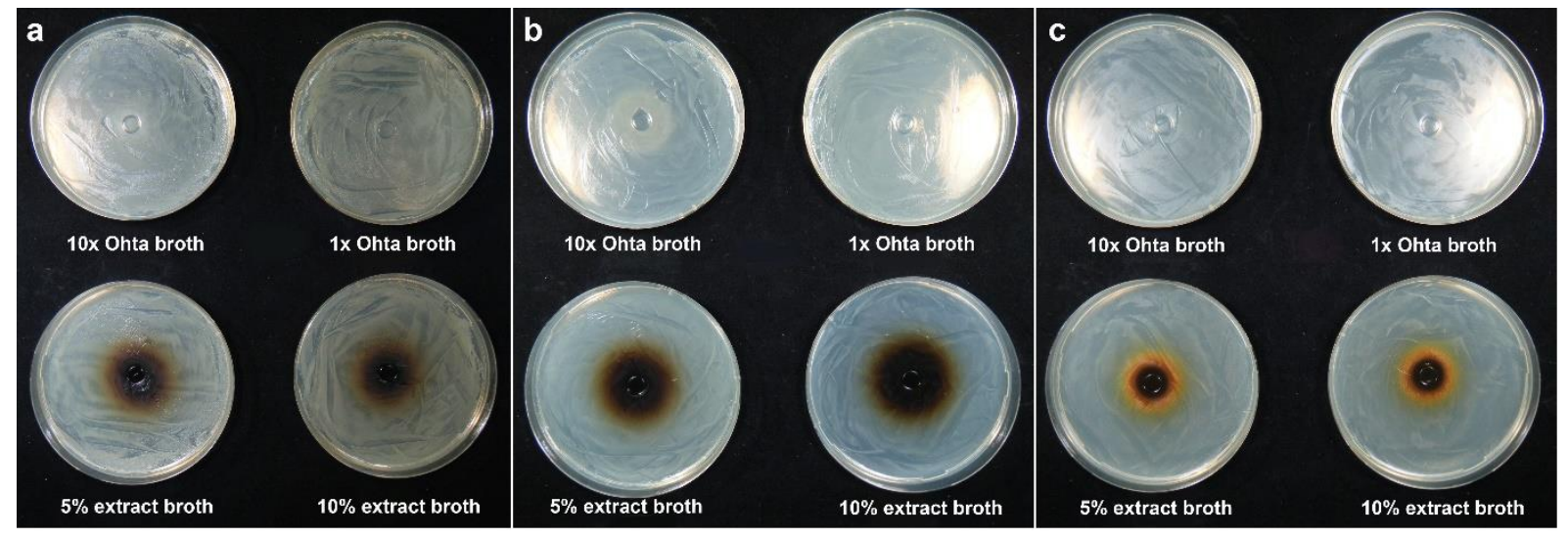

Figure 7. Antibacterial activities of fungal culture broth extract in Gram-negative bacteria.

The inside circle is derived from a contact of peni-cylinder on the surface of the plate; the outer circle indicates the clear zone

Note: a: No effect on the growth of Asaia bogorensis; b: No effect on the growth of Escherichia coli; c: The inhibited effect on growth of Gluconobacter oxydans

\section{Discussion}

Colonies of Ph. spongiosus were invaded and covered by those of $T$. viride. It should be derived from the property of the mycoparasite of $T$. viride (Harman \& Kubicek, 1998). It would be a rare phenomenon in edible mushrooms, i.e. colonies of $P h$. spongiosus inhibited the expansion of $A$. niger colonies and $P$. citrinum colonies, and invaded colonies of both molds. The inhibition potential of $P h$. spongiosus against $A$. niger and $P$. citrinum is suspected to be derived from the mycelial excretions which could have antimicrobial property. The various 
patterns presented in the interaction of $P h$. spongiosus with A. niger and $P$. citrinum was ambiguous.

The inhibit potential of $P h$. spongiosus on soil molds opened the applicability for using it as pathogenic antagonism. Combining with its ectomycorrhizal potential in Citrus spp., this fungus can be applied to increase the harvest of several citrus crops in tropical areas. However, this study should be expanded to investigate the interaction between $P h$. spongiosus and several other plant pathogenic fungi in soil. Moreover, the further studies about the tri-partitions or also tetra-partitions among ectomycorrhizal fungus $P h$. spongiosus, its host plants, plant pathogenic fungi in soil and common soil fungi should be attended for clarifying multi-interaction inside mycorrhizosphere.

The culture broth of $P h$. spongiosus presented the antibacterial activity not only on $\mathrm{Gr}^{+}$ bacteria Bacillus spp. but also on $\mathrm{Gr}^{-}$bacteria $G$. oxydans. The no effect in control tests, even 10x Ohta broth, clearly indicated that the antibacterial activities were derived from the excretion of Ph. spongiosus in stock culture. However, the antibacterial activities look like a weak effect with a small clear zone. It is suggested those activities should be from fungal compounds included in the culture broth. There are many records about the antibacterial activity of fungal extraction from mycelium/sporocarp/culture broth. Most of them are derived from the extract of sporocarps or mycelium. Some are from compounds in sporocarps such as including $\mathrm{S}$ from Omphalotus japonicus syn. Pleurotus japonicus and Clitocybe illuden (Hara, Yoshida, Morimoto, \& Nakano, 1987), and cordycepin from Cordyceps spp. (Sentenac, Ruet, \& Fromageot, 1968). Some are from the extract of vegetative mycelia as the study of Sasek and Musilek (1967) on several ectomycorrhizal fungi but only 4/16 species in this study showed the antibacterial activities. Moreover, the investigation of Alves et al. (2013) showed that phenolic compounds in wild mushrooms had antibacterial activity. In Ph. portentosus and Ph. colosus, phenolic compounds were determined (Kaewnarin, Suwannarach, Kumla, \& Lumyong, 2016; Liaotracoon \& Liaotracoon, 2018). Therefore, exudates of Ph. spongiosus mycelia maybe contain phenolic compounds. These compounds might cause the black color of the cultured medium after ca. 3 weeks.

\section{Conclusion}

Ph. spongiosus colonies were infiltrated and invaded by the mycelia of $T$. viride which is a mycoparasitic fungus. However, this ectomycorrhizal fungus had the deadlock antagonism with a soil fungus $A$. niger and the deadlock competition with another soil fungus $P$. citrinum. Further field studies are required to clarify the interactions of Ph. spongiosus in the mycorrhizosphere of citrus with other organisms such as arbuscular mycorrhizae on Citrus spp., several harmful/profitable soil-born molds, soil fauna (including root aphids), and rhizosphere bacteria (including Bacillus spp.). The screening of antibacterial and antifungal activities of $P h$. spongiosus under in vitro ectomycorrhization is necessary to be conducted in the future.

The biological activity of the broth culture of Ph. spongiosus is another conspicuous characteristic. Culture broth of this fungus showed antibacterial activity on the growth of both Gram-positive and negative bacteria. The edibleness and antibacterial activity remarked this 
fungus to be a candidate for pharmaceutical application. However, additional research is necessary for determining principal components for the activities of culture exudates.

\section{References}

Alves, M. J., Ferreira, I. C. F. R., Froufe, H. J. C., Abreu, R. M. V., Martins, A., \& Pintado, M. (2013). Antimicrobial activity of phenolic compounds identified in wild mushrooms, SAR analysis and docking studies. Journal of Applied Microbiology, 115(2), 346-357.

Chang, S. T., \& Miles, P. G. (2004). Mushrooms cultivation, nutritional value, medicinal effect, and environmental impact (2nd ed.). Boca Raton, FL: CRC Press LLC.

Cooke, R. C., \& Rayner, A. D. M. (1984). Ecoloy of saprotrophic fungi. New York, NY: Longman Inc.

Cooke, R. C., \& Whipps, J. M. (1993). Ecophysiology of fungi. Oxford, UK: Blackwell Scientific Publication.

Frankland, J. C., Hedger, J. N., \& Swift, M. J. (1982). Decomposer basidiomycetes: Their biology and ecology. Cambridge, UK: Cambridge University Press.

Hara, M., Yoshida, M., Morimoto, H., \& Nakano, H. (1987). 6-Deoxyilludin M, a new antitumor antibiotic: Fermentation, isolation and structural identification. The Journal of Antibiotics, 40(11), 1643-1646.

Harman, G. E., \& Kubicek, C. P. (1998). Trichoderma and Gliocladium, volume 2: Enzymes, biological control and commercial applications. London, UK: Taylor and Francis Ltd.

Kaewnarin, K., Suwannarach, N., Kumla, J., \& Lumyong, S. (2016). Phenolic profile of various wild edible mushroom extracts from Thailand and their antioxidant properties, antityrosinase and hyperglycaemic inhibitory activities. Journal of Functional Foods, 27, $352-364$.

Liaotracoon, W., \& Liaotracoon, V. (2018). Influence of drying process on total phenolics, antioxidative activity and selected physical properties of edible bolete (Phlebopus colossus (R. Heim) Singer) and changes during storage. Journal of Food Science and Technology, Campinas, 38(2), 231-237.

Marx, D. H. (1969). The influence of ectotrophic mycorrhizal fungi on the resistance of pine roots to pathogenic infections. I. Antagonism of mycorrhizal fungi to root pathogenic fungi and soil bacteria. Phytopathology, 59, 153-163.

Ohshima, T. (2011). Various functionality of ergothioneine in fruiting bodies and solid cultivating media of mushrooms - meat color stabilization, antioxidative stress, skin whitening. In Proceeding of the $15^{\text {th }}$ annual meeting of Japanese Society of Mushroom Science and Biotechnology (pp. 20-21).

Ohta, A. (1990). A new medium for mycelial growth of mycorrhizal fungi. Transactions of the Mycological Society of Japan, 31(3), 323-334.

Pham, N. D. H., Takahashi, H., Fukiharu, T., Shimizu, K., Le, B. D., \& Suzuki, A. (2012a). Phlebopus spongiosus sp. nov. (Boletales, Boletinellaceae) with a sponge-like tissue. Mycotaxon, 119(1), 27-34. doi:10.5248/119.27 
Pham, N. D. H., Yamada, A., Shimizu, K., Noda, K., Dang, L. A. T., \& Suzuki, A. (2012b). A sheathing mycorrhiza between the tropical bolete Phlebopus spongiosus and Citrus maxima. Mycoscience, 53(5), 347-353. doi:10.1007/S10267-011-0177-5

Rutter, G. (2010). Fungi and humanity. In L. Boddy \& M. Colemen (Eds.), From another kingdom the amazing world of fungi. Edinburgh, UK: Royal Botanic garden Edinburgh.

Sasek, V., \& Musilek, V. (1967). Cultivation and antibiotic activity of mycorrhizal basidiomycetes. Folia Microbiologica, 12(6), 515-523.

Sentenac, A., Ruet, A., \& Fromageot, P. (1968). Excision de la region du DNA liee a la RNA polymerase in vitro. Federation of European Biochemical Societies Letters, 2(1), 53-56.

Stamets, P. (2005). Mycelium running: How mushrooms can help save the world. Berkeley, CA: Ten Speed Press.

Wainwright, M. (2010). Amazing chemists. In L. Boddy \& M. Colemen (Eds.), From another kingdom the amazing world of fungi. Edinburgh, UK: Royal Botanic garden Edinburgh.

Woodland, S., \& Boddy, L. (2008). Interactions between saprotrophic fungi. In L. Boddy, J. C. Frankland \& P. Van West (Eds.), Ecology of saprotrophic basidiomycetes. London, UK: Academic Press. 